\title{
Electron transport in a ferromagnetic/normal/ferromagnetic tunnel junction based on the surface of a topological insulator
}

\author{
Jian-Hui Yuan* and Yan Zhang \\ The department of Physics, Guangxi medical university, Nanning, Guangxi, 530021, China \\ Jian-Jun Zhang and Ze Cheng \\ School of Physics, Huazhong University of Science and Technology, Wuhan 430074, China
}

(Dated: September 12, 2018)

\begin{abstract}
We theoretically study the electron transport properties in a ferromagnetic/normal/ferromagnetic tunnel junction, which is deposited on the top of a topological surface. The conductance at the parallel (P) configuration can be much bigger than that at the antiparallel (AP) configuration. Compared $\mathbf{P}$ with $\mathbf{A P}$ configuration, there exists a shift of phase which can be tuned by gate voltage. We find that the exchange field weakly affects the conductance of carriers for $\mathbf{P}$ configuration but can dramatically suppress the conductance of carriers for AP configuration. This controllable electron transport implies anomalous magnetoresistance in this topological spin valve, which may contribute to the development of spintronics . In addition, we find that there is a Fabry-Perot-like electron interference.

PACS numbers: $72.80 . S k, 73.40 .-\mathrm{c}, 75.50 . \mathrm{Gg}$

Keywords: Topological insulator, Electronic transport, Ferrimagnetic
\end{abstract}




\section{INTRODUCTION}

The concept of a topological insulator (TI) dates back to the work of Kane and Mele, who focused on two-dimensional (2D) systems ${ }^{1}$. There has been much recent interest in TIs, threedimensional insulators with metallic surface states protected by time reversal invariance $[1-25]$. Its theoretical [2] and experimental [3] discovery has accordingly generated a great deal of excitement in the condensed matter physics community. In particular, the surface of a three-dimensional (3D) TI, such as $\mathrm{Bi}_{2} \mathrm{Se}_{3}$ or $\mathrm{Bi}_{2} \mathrm{Te}_{3}$ [4], is a $2 \mathrm{D}$ metal, whose band structure consists of an odd number of Dirac cones, centered at time reversal invariant momenta in the surface Brillouin zone [5]. This corresponds to the infinite mass Rashba model [6], where only one of the spin-split bands exists. This has been beautifully demonstrated by the spin- and angle-resolved photoemission spectroscopy $[7,8]$. Surface sensitive experiments such as angle-resolved photoemission spectroscopy (ARPES) and scanning tunneling microscopy (STM) $[9,10]$ have confirmed the existence of this exotic surface metal, in its simplest form, which takes a single Dirac dispersion. Recent theoretical and experimental discovery of the two dimensional (2D) quantum spin Hall system [11 - 18] and its generalization to the $\mathbf{T I}$ in three dimensions $[19-21]$ have established the state of matter in the time-reversal symmetric systems.

The time-reversal invariant $\mathbf{T I}$ is a new state of matter, distinguished from a regular band insulator by a nontrivial topological invariant, which characterizes its band structure [11]. Currently, most works focus on searching for TI materials and novel transport properties. To my knowledge, the fabrication of such TI-based nanostructure is still a challenging task. Usually such structures are fabricated by utilizing the split gate and etching technique [22]. On the other hand, the 3D TIs are expected to show several unique properties when the time reversal symmetry is broken [23 - 25]. This can be realized directly by a ferromagnetic insulating (FI) layer attached to the 3D TI surface. One remarkable feature of the Dirac fermions is that the Zeeman field acts like a vector potential: the Dirac Hamiltonian is transformed as $\sigma \cdot \mathbf{k} \longrightarrow \sigma \cdot(\mathbf{k}+\mathbf{H})$ by the Zeeman field $\mathbf{H}$ [26]. This is in contrast to the Schrödinger electrons in conventional semiconductor heterostructures modulated by nanomagnets [27 - 29].

In this work, we study the electron transport properties in a ferromagnetic $(\mathbf{F}) /$ normal/N)/ ferromagnetic (F) tunnel junction, which is deposited on the top of a topological surface. Ferromagnetic 
Permalloy electrodes are formed by electron-beam lithography (EBL) followed by thermal evaporation; a second EBL step establishes contact to the Permalloy via $\mathrm{Cr} / \mathrm{Au}$ electrodes [30]. As shown in Fig.1, the FI is put on the top of the TI to induce an exchange field via the magnetic proximity effect. The easy axis of a FI stripe is usually along its length direction and thus either in parallel (P) or antiparallel (AP) with the $+y$ axis. We find that the conductance at the $\mathbf{P}$ configuration can be much bigger than that at the AP configuration. Compared $\mathbf{P}$ with $\mathbf{A P}$ configuration, there exists a shift of phase which can be tuned by gate voltage. We find that the exchange field weakly affects the conductance of carriers for $\mathbf{P}$ configuration but can dramatically suppress the conductance of carriers for AP configuration. This controllable electron transport implies anomalous magnetoresistance in this topological spin valve, which may contribute to the development of spintronics. Compared with the conventional F/N/F tunneling based on two dimensional electron gas (2DEG), the result implies the existence of Fabry-Perot-like electron interference in F/N/F based on the TI. In Sec. II , we introduce the model and method for our calculation. In Sec. III, the numerical analysis to our important issues is reported. Finally, a brief summary is given in sec. IV.

\section{MODEL AND METHOD}

Now, let us consider a $\mathbf{F} / \mathbf{N} / \mathbf{F}$ tunnel junction which is deposited on the top of a topological surface where a gate electrode is attached to the ferromagnetic material. The ferromagnetism is induced due to the proximity effect by the ferromagnetic insulators deposited on the top as shown in Fig. 1. We assume that the initial magnetization of FI stripes in the region I is aligned with the $+y$ axis. In an actual experiment, one can use a magnet with very strong (soft) easy axis anisotropy to control the ferromagnetic material. Thus we focus on charge transport at the Fermi level of the surface of TIs, which is described by the 2D Dirac Hamiltonian

$$
H=v_{F} \sigma \cdot \mathbf{p}+\sigma \cdot \mathbf{M}+V(x)
$$

where $\sigma$ is Pauli matrices, $\mathbf{M}=M_{y}(x)=M_{0}(\Theta(-x)+\gamma \Theta(x-L))$ is the effective exchange field and $V(x)=U_{g} \Theta(x) \Theta(L-x)+V_{g} \Theta(x-L)$ is the gate voltage, where $\gamma=+1(-1)$ corresponds to the $\mathbf{P}(\mathbf{A P})$ configurations of magnetization and $\Theta(x)$ is the Heaviside step function. 

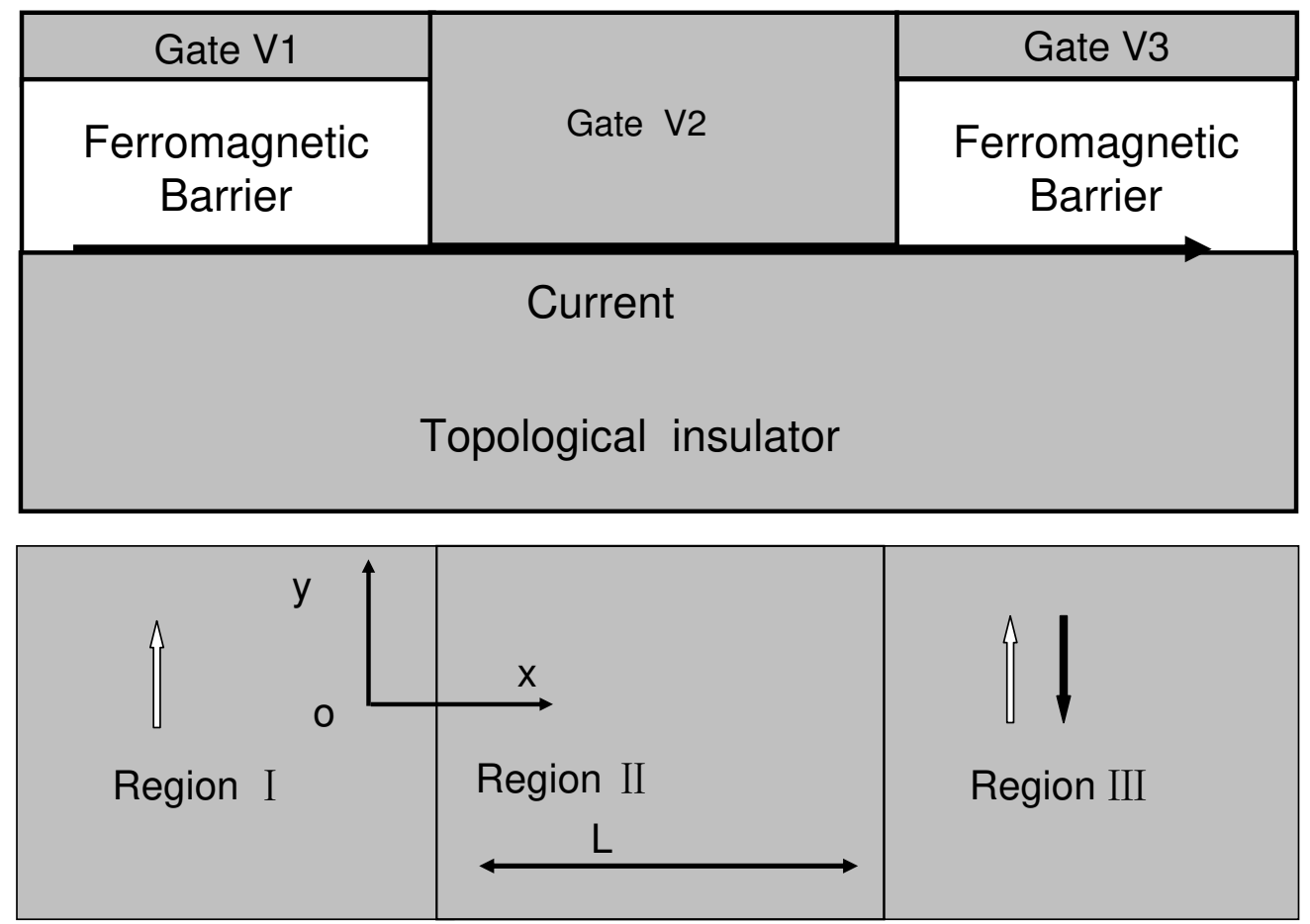

FIG. 1: Schematic illustration of the device. Top: Schematic diagram of two ferromagnetic barriers on the topological surface divided by a gate electrode at a distance $L$. Bottom: The magnetization directions of adjacent $\mathbf{F I}$ stripes are parallel $(\mathbf{P})$ in the configuration and antiparallel (AP) in the configuration.

Because of the translational invariance of the system along $y$ direction, the equation $H \Psi(x, y)=$ $E \Psi(x, y)$ admits solutions of the form $\Psi(x, y)=\left(\Psi_{1}(x), \Psi_{2}(x)\right)^{T} \exp \left(i k_{y} y\right)$. We set $\hbar=v_{F}=1$ in the following. Then, with the above Hamiltonian, the wave function in the whole system is given by

$$
\Psi_{1}= \begin{cases}\exp \left(i k_{x_{1}} x\right)+r \exp \left(-i k_{x_{1}} x\right), & x<0, \\ a \exp \left(i q_{x} x\right)+b \exp \left(-i q_{x} x\right), & 0<x<L, \\ t \exp \left(i k_{x_{2}}(x-L)\right), & x>L,\end{cases}
$$




$$
\Psi_{2}= \begin{cases}\alpha^{+} \exp \left(i k_{x_{1}} x\right)+r \alpha^{-} \exp \left(-i k_{x_{1}} x\right), & x<0, \\ a \beta^{+} \exp \left(i q_{x} x\right)+b \beta^{-} \exp \left(-i q_{x} x\right), & 0<x<L, \\ t \alpha \exp \left(i k_{x_{2}}(x-L)\right), & x>L,\end{cases}
$$

where $k_{x_{1}}=E \cos \theta_{F_{1}}, q_{x}=\left(E-U_{g}\right) \cos \theta$ and $k_{x_{2}}=\left(E-V_{g}\right) \cos \theta_{F_{2}}$ are wave vectors in region I, region II and region III, $\alpha^{ \pm}= \pm \exp \left( \pm i \theta_{F_{1}}\right), \beta^{ \pm}= \pm \exp ( \pm i \theta)$ and $\alpha=\exp \left(i \theta_{F_{2}}\right)$. The momentum $k_{y}$ conservation should be satisfied everywhere such as $k_{y}=E \sin \theta_{F_{1}}-M_{0}=\left(E-U_{g}\right) \sin \theta=$ $\left(E-V_{g}\right) \sin \theta_{F_{2}}-\gamma M_{0}$. Also, $r$ and $t$ are reflection and transmission coefficients, respectively. Continuities of the wave function $\Psi$ at $x=0$ and $x=L$ are $\Psi\left(0^{-}\right)=\Psi\left(0^{+}\right)$and $\Psi\left(L^{-}\right)=\Psi\left(L^{+}\right)$, respectively. We find that the transmitted electron coefficient $t_{\gamma}$ is given by

$$
t_{\gamma}=\frac{2 \cos \theta_{F_{1}} \cos \theta \exp \left(-i k_{x_{2}} L\right)}{s_{1, \gamma} \cos \left(q_{x} L\right)+i s_{2, \gamma} \sin \left(q_{x} L\right)},
$$

with $s_{1, \gamma}=\cos \theta\left(\exp \left(i \theta_{F_{2}}\right)+\exp \left(-i \theta_{F_{1}}\right)\right)$ and $s_{2, \gamma}=i \sin \theta\left(\exp \left(-i \theta_{F_{1}}\right)-\exp \left(i \theta_{F_{2}}\right)\right)-\exp \left(i\left(\theta_{F_{2}}-\right.\right.$ $\left.\left.\theta_{F_{1}}\right)\right)-1$. Then

$$
T_{\gamma}=\left|t_{\gamma}\right|^{2} \Re\left(\cos \theta_{F_{2}} / \cos \theta_{F_{1}}\right),
$$

where the factor $\Re\left(\cos \theta_{F_{2}} / \cos \theta_{F_{1}}\right)$ is due to current conservation. In the linear transport regime and for low temperature, we can obtain the conductance $G$ by introducing it as the electron flow averaged over half the Fermi surface from the well-known Landauer-Buttiker formula $[25,31,32]$

$$
G_{\gamma} \sim 1 / 2 \int_{-\pi / 2}^{\pi / 2} T_{\gamma}\left(E_{F}, E_{F} \cos \theta_{F_{1}}\right) \cos \theta_{F_{1}} d \theta_{F_{1}}
$$

\section{RESULTS AND DISCUSSIONS}

For convenience we express all quantities in dimensionless units by means of the length of the basic unit $L$ and the energy $E_{0}=\hbar v_{F} / L$. For a typical value of $L=50 \mathrm{~nm}$ and the $\mathrm{Bi}_{2} \mathrm{Se}_{3}$ material $v_{F}=5 \times 10^{5} \mathrm{~m} / \mathrm{s}$, one has $E_{0}=6.6 \mathrm{meV}$. We set the energy of electron $E=E_{F}$ and also define the value $\eta$ with the form $\eta=M_{0} / E_{F}$ in our calculation. 

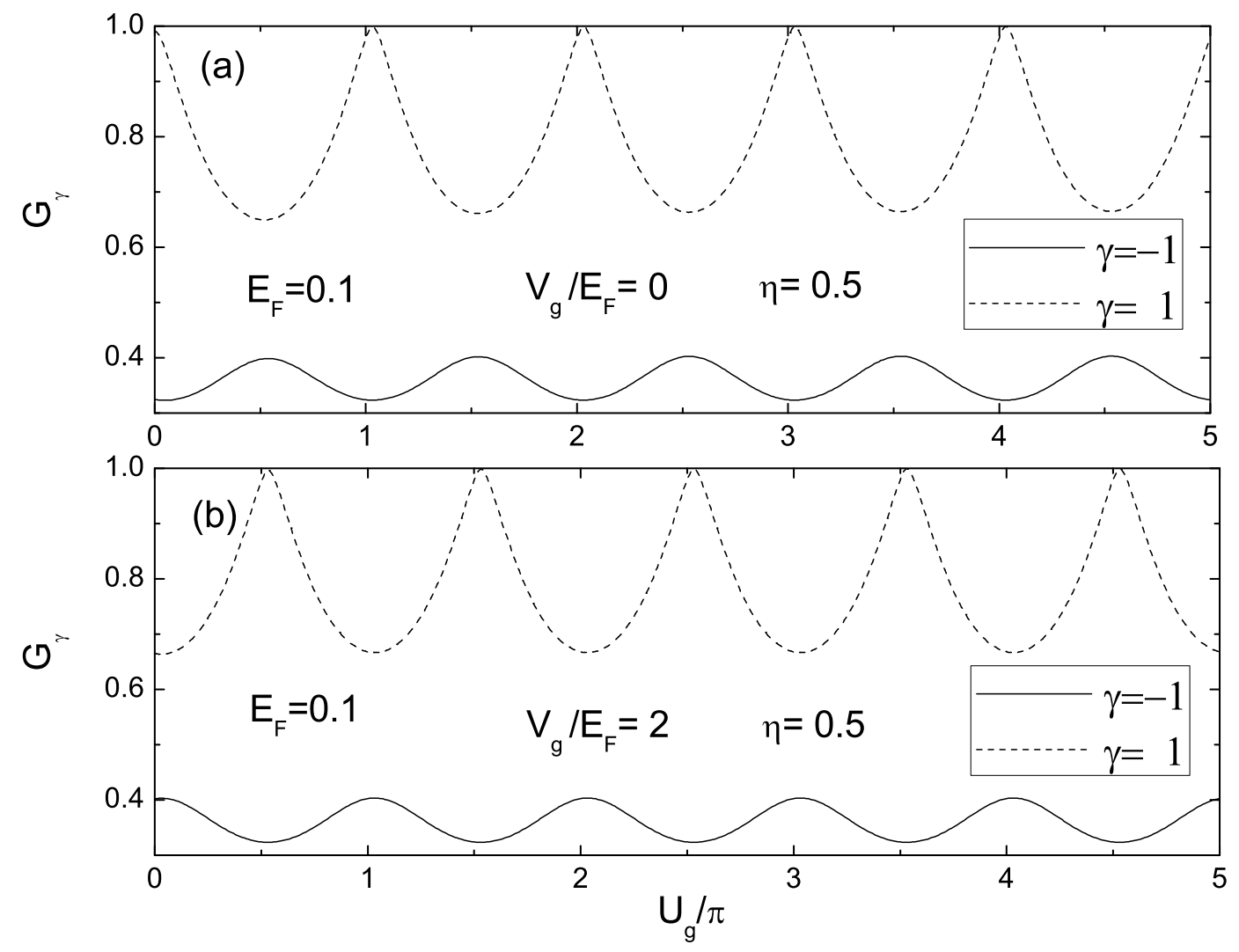

FIG. 2: Gate voltage dependence of the conductances with a $\mathbf{P}(\gamma=1)$ and $\mathbf{A P}(\gamma=-1)$ configuration in the two cases: (a) $V_{g} / E_{F}=0$ and (b) $V_{g} / E_{F}=2$. The values of the other parameters are $E_{F}=0.1$ and $\eta=0.5$

In Fig.2, we show gate voltage dependence of the conductances with a $\mathbf{P}(\gamma=1)$ and $\mathbf{A P}$ $(\gamma=-1)$ configuration in the two cases: (a) $V_{g} / E_{F}=0$ and (b) $V_{g} / E_{F}=2$. The value of the other parameter is $E_{F}=0.1$ and $\eta=0.5$. The presence of quantum modulation are seen in these two figures. We can see an oscillation of the electrical conductance with a period of $\pi$ when the voltage $U_{g}$ is larger than $E_{F}$. The conductance at the $\mathbf{P}$ configuration can be much bigger than that at the AP configuration. We find that a minimum of conductance at the $\mathbf{P}$ configuration corresponds to a maximum of conductance at the AP configuration [see in fig.2 (a)] when the voltage $U_{g}$ is larger than $E_{F}$. In Fig. 2(b), a similar tendency to Fig. 2(a) is seen. In distinct contrast to Fig.2(a), a minimum of conductance at the $\mathbf{P}$ configuration here corresponds to a maximum of conductance 
at the AP configuration [see in fig.2 (b)]. That is to say, there exists a shift of $\pi$-phase. To understand these results intuitively, we consider that the gate voltage $U_{g}$ is larger than the Fermi energy $E_{F}$. For the given Fermi energy $E_{F}=0.1$, the condition $U_{g} \gg E_{F}$ is easily satisfied. In this limit we have $\theta \rightarrow 0$ and hence the transmission probability $T_{\gamma} \sim\left(2 \cos ^{2} \theta_{F_{1}} /\left(1+\cos \theta_{F_{1}} \cos \theta_{F_{2}}-\right.\right.$ $\left.\cos \left(2 U_{g} L\right) \sin \theta_{F_{1}} \sin \theta_{F_{2}}\right) \Re\left(\cos \theta_{F_{2}} / \cos \theta_{F_{1}}\right)$. For $\gamma=1$ and $V_{g} / E_{F}=0$ (or 2), we find the $\theta_{F_{1}} \equiv \theta_{F_{2}}$ (or $-\theta_{F_{2}}$ ), and thus $T_{\gamma} \sim \cos ^{2} \theta_{F_{1}} /\left(1-\cos ^{2}\left(U_{g} L+\delta\right) \sin ^{2} \theta_{F_{1}}\right)$ where $\delta=0$ (or $\pi / 2$ ) corresponds to $V_{g} / E_{F}=0$ (or 2). Thus the phase difference between $V_{g} / E_{F}=0$ and $V_{g} / E_{F}=2$ is given by $U_{g} L$. We find $G_{\gamma} \propto \cos ^{2}\left(U_{g} L\right)$ for $V_{g} / E_{F}=0$ but $G_{\gamma} \propto \sin ^{2}\left(U_{g} L\right)$ for $V_{g} / E_{F}=2$. When $U_{g} L$ is equal to the half period of $\pi$, a minimum of conductance will appear for $V_{g} / E_{F}=0$ but a maximum of conductance will appear for $V_{g} / E_{F}=2$. When $U_{g} L$ is equal to the period of $\pi$, a maximum of conductance will appear for $V_{g} / E_{F}=0$ but a minimum of conductance will appear for $V_{g} / E_{F}=2$. Furthermore, we find that $G_{\gamma}$ oscillates between $2 / 3$ and 1 for $\gamma=1$. For $\gamma=-1$ and $V_{g} / E_{F}=0$ (or 2 ), there is a similar tendency to the case of $\gamma=1$. We can see that $G_{\gamma}$ is suppressed obviously by the strength of the effective exchange field . Nevertheless, there exists a shift of $\pi$-phase because of the factor $\cos \left(2 U_{g} L\right)$.

In order to observe the effect of the exchange field $\eta$ on the conductance, in Fig. 3 we show the gate voltage dependence of the conductances with a $\mathbf{P}(\gamma=1)$ and $\mathbf{A P}(\gamma=-1)$ configuration for four different values $\eta=0,0.2,0.5$, and 0.8 . The solid lines are for $V_{g} / E_{F}=0$ while the dashed lines are for $V_{g} / E_{F}=2$. The value of the other parameter is $E_{F}=0.1$. A similar tendency to Fig. 2 is seen in Fig. 3. It is easily seen that the exchange field $\eta$ weakly affects the conductance of carriers for $\gamma=1$ but profoundly influences the conductance of carriers for $\gamma=-1$. For $\gamma=-1$, $G_{\gamma}$ is suppressed obviously by increasing the value $\eta$. Due to current conservation, the factor $\Re\left(\cos \theta_{F_{2}} / \cos \theta_{F_{1}}\right)$ must be real and then we have $\sin \theta_{F_{1}}= \pm \sin \theta_{F_{2}}+2 \eta$ where sign + (or -) corresponds to $V_{g} / E_{F}=0$ (or 2 ). We can see $2 \eta-1 \leq \sin \theta_{F_{1}} \leq 1$ and $2 \eta-1 \leq \sin \left(\mp \theta_{F_{2}}\right) \leq 1$ where sign - (or + ) corresponds to $V_{g} / E_{F}=0$ (or 2). Thus we find the ranges of the angleallowable $\theta_{F_{1}}$ and $\theta_{F_{2}}$ depend on $\eta$. The transmission is nonzero only for $\theta_{F_{1}}$ and $\theta_{F_{2}}$ in these ranges and vanishes for $\eta \geq 1$. The number of channels decreases with increasing of $\eta$, so we can see that $G_{\gamma}$ dramatically decreases with the increase of $\eta$ for $\gamma=-1$. Noting that the $\eta \geq 1$ for $\gamma=-1$, the conductance of carriers is forbidden, which implies anomalous magnetoresistance in this topological spin valve. 


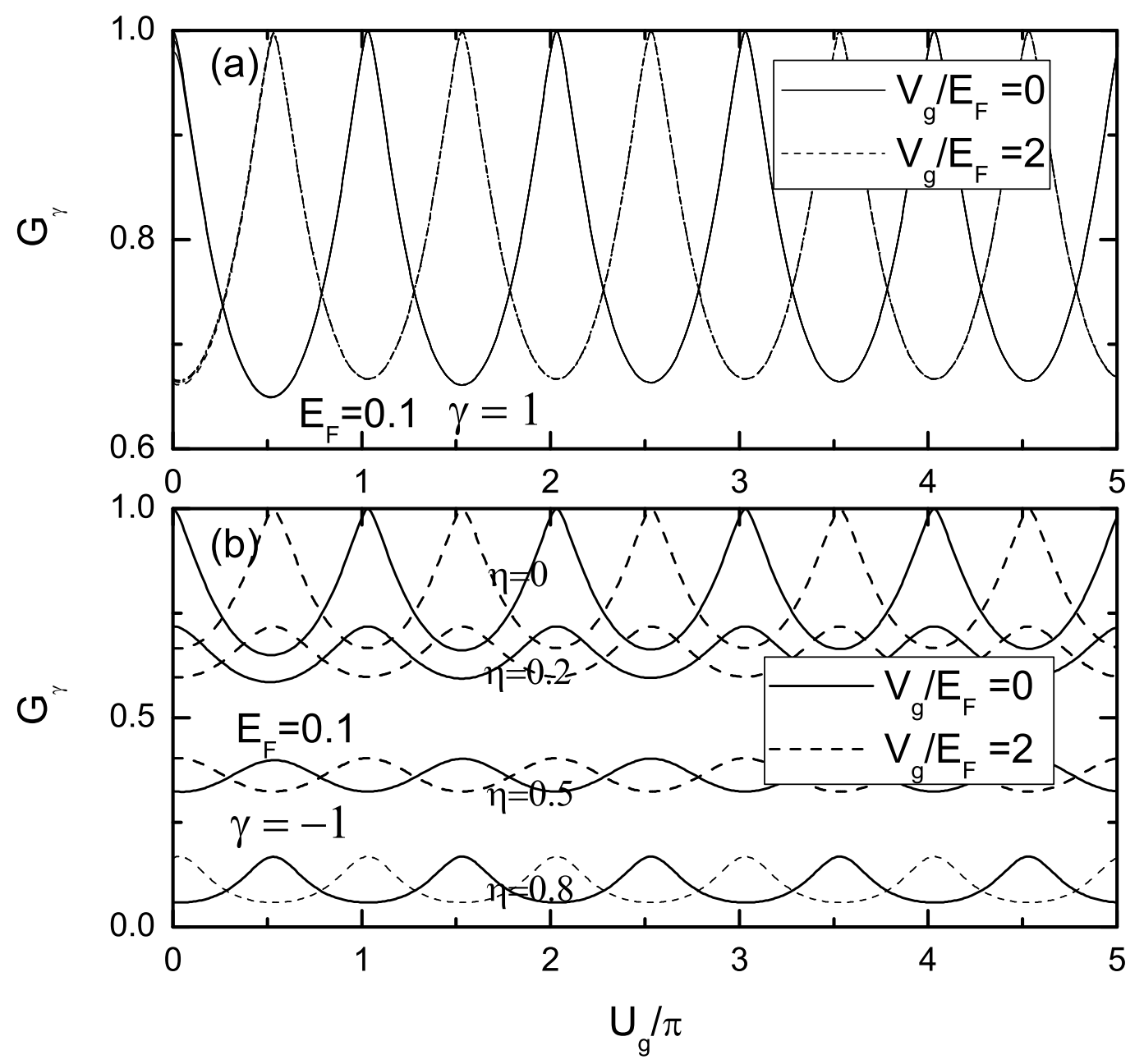

FIG. 3: Gate voltage dependence of the conductances with a $\mathbf{P}(\gamma=1)$ and $\mathbf{A P}(\gamma=-1)$ configuration for four different values $\eta=0,0.2,0.5$, and 0.8 . The solid lines are for $V_{g} / E_{F}=0$ while the dashed lines are for $V_{g} / E_{F}=2$. The value of the other parameter is $E_{F}=0.1$.

In Fig. 4, we show the gate voltage dependence of the conductances with a $\mathbf{P}(\gamma=1)$ and $\mathbf{A P}(\gamma=-1)$ configuration for three different values $E_{F}=0.1,1.0$, and 5.0. In (a) and (b), the $V_{g}$ is set as $V_{g} / E_{F}=0$ while in (c) and (d) the $V_{g}$ is set as $V_{g} / E_{F}=2$. The value of the other parameter is $\eta=0.5$. For $E_{F}=0.1$, we can see that the $\pi$ periodicity appears. However, the $\pi$ periodicity is broken for $E_{F}=1$ (or 5) because the condition $U_{g} \gg E_{F}$ is not satisfied for the smaller $U_{g}$. Nevertheless, we get the $\pi$ periodicity of conductance again by choosing a bigger $U_{g}$ 

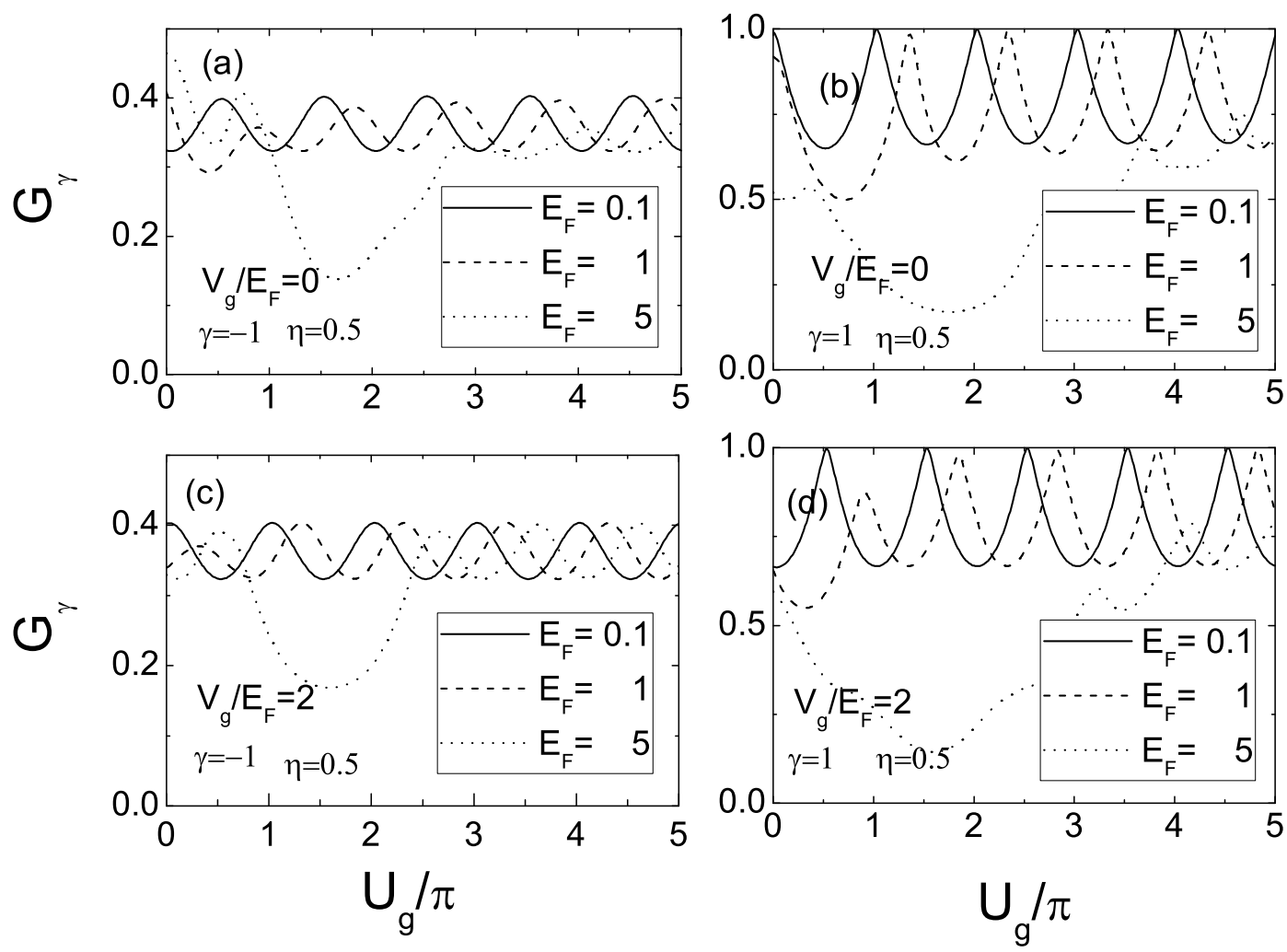

FIG. 4: Gate voltage dependence of the conductances with a $\mathbf{P}(\gamma=1)$ and $\mathbf{A P}(\gamma=-1)$ configuration for three different values $E_{F}=0.1,1.0$, and 5.0. In (a) and (b), the $V_{g}$ is set as $V_{g} / E_{F}=0$ while in (c) and (d) the $V_{g}$ is set as $V_{g} / E_{F}=2$. The value of the other parameter is $\eta=0.5$.

for the bigger $E_{F}$. Furthermore, we find that the minimum of the conductance will appear when the gate voltage arrives at a certain value. It is easily seen that the minimum of the conductance shifts to the right with increasing of the Fermi energy. The larger the Fermi energy is, the smaller the minimum of the conductance is. This phenomena is very obvious for the $\mathbf{P}(\gamma=1)$ configuration [see in figs.4 (a) and (d)]. From Figs.4 and 5, we find that the conductance at the parallel (P) configuration can be much bigger than that at the antiparallel (AP) configuration. However it may be not satisfied for the larger Fermi energy when the gate voltage is not bigger enough. We find that there is a Fabry-Perot-like electron interference in the $\mathbf{F} / \mathbf{N} / \mathbf{F}$ tunnel junction, which is deposited on the top of a topological surface. The two ferromagnetic electrodes and the barrier can compose a 
Fabry-Perot resonator $[33,34]$. The transmitted electron waves in this resonator can be reflected by the two ferromagnetic electrodes. The electron waves undergo multiple reflections back and forth along the resonator between the two ferromagnetic electrodes. The conductance oscillations are caused by the interference of electron waves among the modes of the channel-allowable. When the gate voltage $U_{g}$ is larger than the Fermi energy $E_{F}$, the round trip between the two ferromagnetic electrodes adds a further phase change $\delta \sim 4 \pi / \lambda$ where the Fermi wavelength $\lambda \sim 2 \pi / U_{g}$ because of the value $\theta \sim 0$. When the round trip between the two ferromagnetic electrodes is equal to the a multiple of wavelength, the quantum interference happens. This implies that the oscillation period is equal to $\triangle U_{g}=\pi$.

In order to investigate the solution of the standard electron described by the Schrödinger equation with a parabolic band structure, we consider a 2DEG in $(\mathrm{x}, \mathrm{y})$ plane with a magnetic field $\mathrm{B}$ in the $\mathrm{z}$ direction as described in Refs.[26-28]. Thus we can fabricate a F/N/F tunneling based on the 2DEG. We can apply all the relevant quantities in dimensionless units, which are the same with the Ref. 28. So we can define the value $\Delta=M_{0}=B$ where $M_{0}$ is the the effective exchange field corresponding to a $\mathbf{F} / \mathbf{N} / \mathbf{F}$ tunneling based on the TI and $B$ is the magnetic field corresponding to a F/N/F tunneling based on 2DEG. Nevertheless, we ignore the splitting of energy induced by the spin of electron. As described in Fig.1, we set the left electrode potential $V_{1}=0$. For the electron with parabolic spectrum, $E_{F} \sin \theta_{F_{1}}$ in Eq.(6) should be replaced by $\sqrt{2 E_{F}} \sin \theta_{F_{1}}$. Then the continuity of the wave function gives the transmission coefficient

$$
t_{\gamma}=\frac{2 k_{1} q_{x}}{-q_{x}\left(k_{1}+k_{3}\right) \cos \left(q_{x} L\right)+i\left(k_{1} k_{3}+q_{x}^{2}\right) \sin \left(q_{x} L\right)},
$$

and transmission probability $T_{\gamma}=\left|t_{\gamma}\right|^{2} \mathfrak{R}\left(k_{3} / k_{1}\right)$ where $k_{1}=\sqrt{2\left(E_{F}-V_{1}\right)} \cos \theta_{F_{1}}, q_{x}=$ $\sqrt{2\left(E_{F}-V_{2}\right)} \cos \theta$ and $k_{3}=\sqrt{2\left(E_{F}-V_{3}\right)} \cos \theta_{F_{3}}$. We can easily see that $T_{\gamma} \equiv 0$ when the Fermi energy is smaller than the right electrode voltage $V_{3}$, which is different from the case of Dirac band structure. In Fig.6, we show the wave vector $k_{y}$ dependence of the transmission probability with a $\mathbf{P}(\gamma=1)$ and $\mathbf{A P}(\gamma=-1)$ configuration for Dirac electrons shown in (a) and the standard electrons shown in (b). The values of the other parameter are $\Delta=0.5, V_{2}=U_{g}=0$, $V_{3}=V_{g}$ and $k_{x}=k_{x_{1}}=k_{1}=2$. We can find that transmission is significantly more pronounced for Dirac electrons than for the usual electrons. Compared with the $\mathbf{P}(\gamma=1)$ configuration, it can be seen that the channel of electron transporting from the left electrode to the right electron 

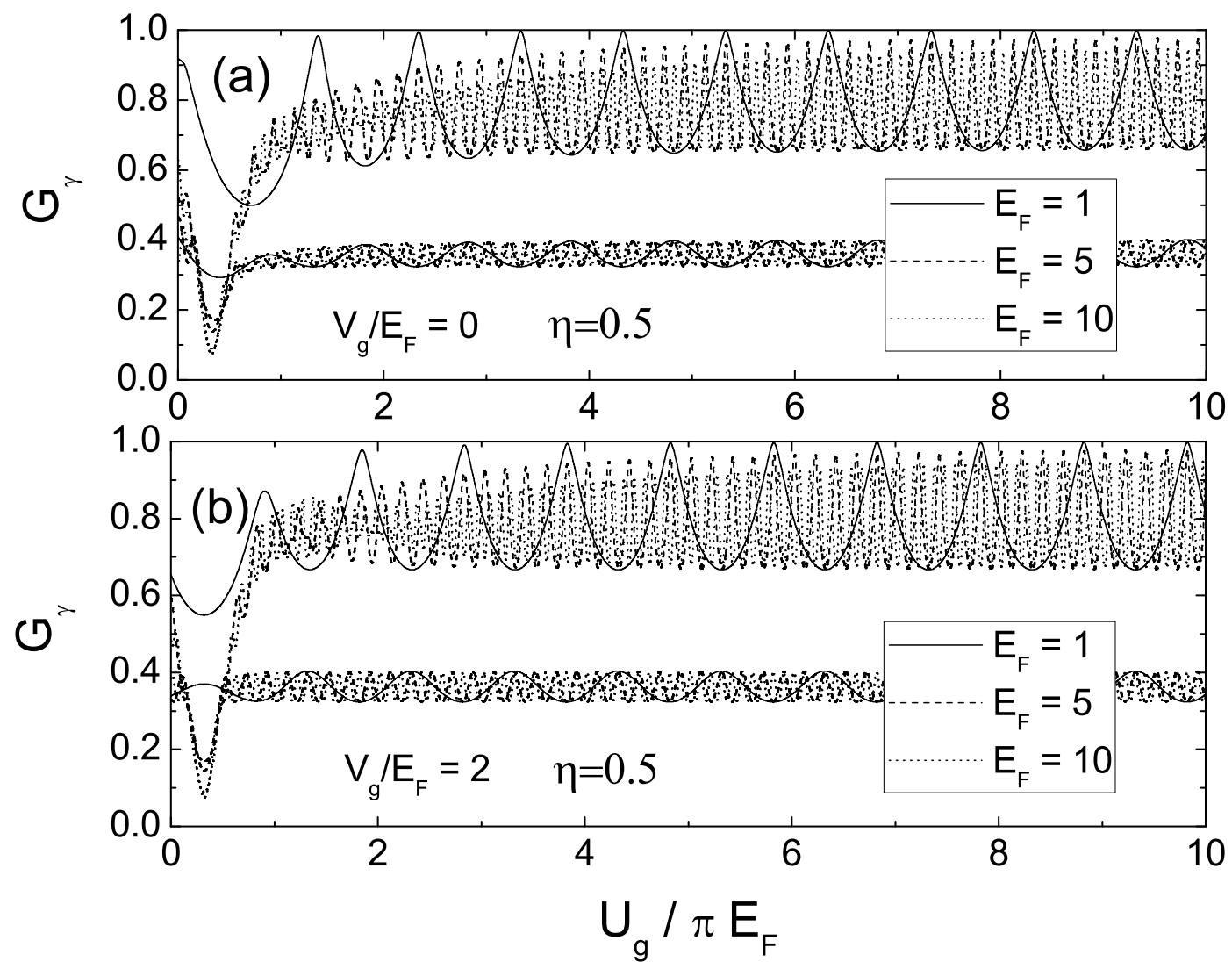

FIG. 5: Gate voltage dependence of the conductances with a $\mathbf{P}(\gamma=1)$ and $\mathbf{A P}(\gamma=-1)$ configuration for three different values $E_{F}=1.0,5.0$, and 10.0. In (a), the $V_{g}$ is set as $V_{g} / E_{F}=0$ while in (b) $V_{g}$ is set as $V_{g} / E_{F}=2$. The value of the other parameter is $\eta=0.5$.

is suppressed especially for the standard electron. It is easily seen that the variety of the tunneling conductance of $\mathrm{F} / \mathrm{N} / \mathrm{F}$ with the change of the gate voltage is obviously different between the Dirac electron and standard electron [see in Fig.7]. For the standard electron, the conductance will decrease monotonously with the increase of the gate voltage because of evanescent wave modes. However, we can see that the conductance with a $\pi$ periodicity appears as the gate voltage is large enough for the Dirac electron. On the one hand, Dirac confined electron exhibits a jittering motion called Zitterbewegung, originating from the interference of states with positive and negative energy. On the other hand, the transmitted Dirac electron waves in this resonator can be reflected 

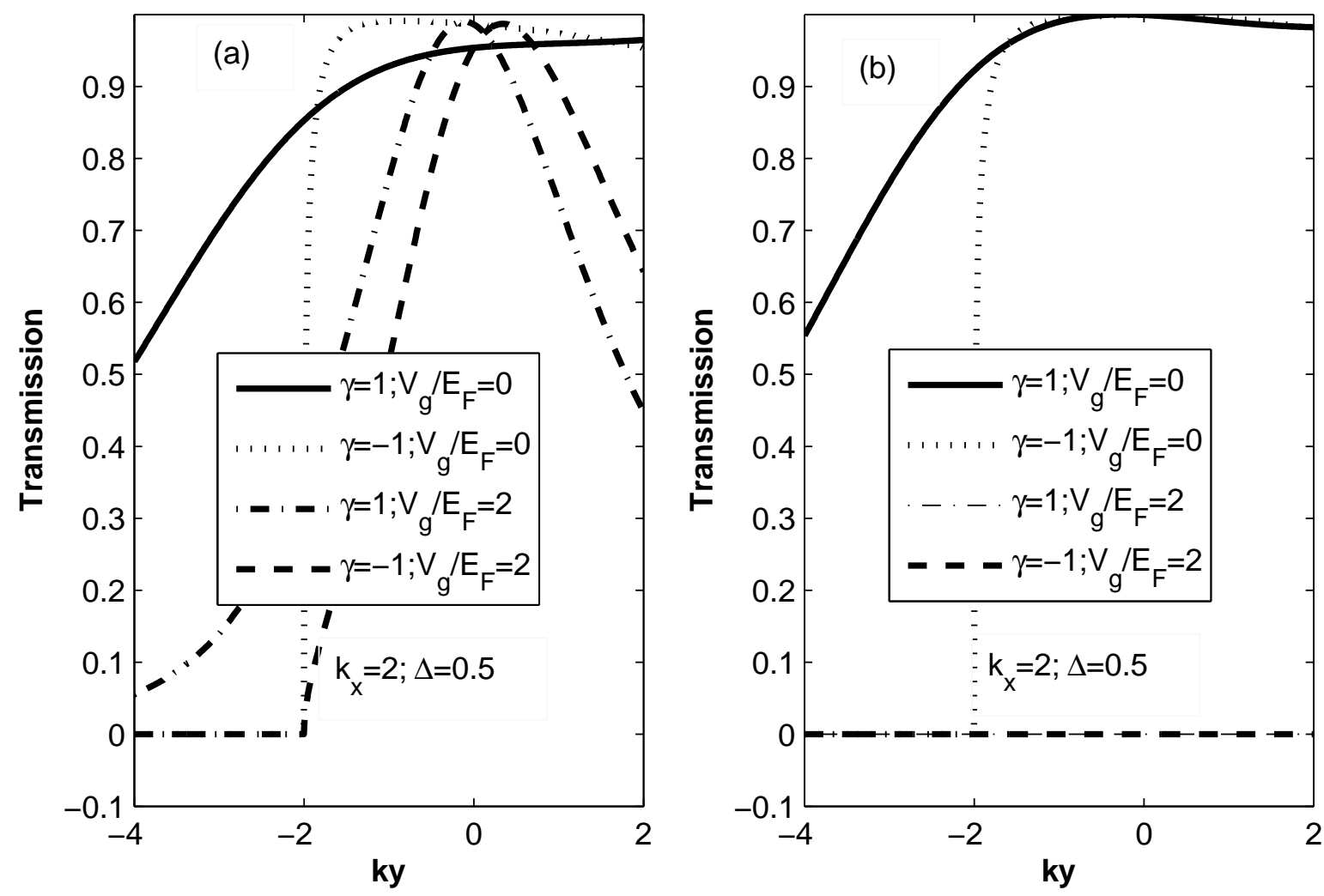

FIG. 6: Wave vector $k_{y}$ dependence of the transmission probability with a $\mathbf{P}(\gamma=1)$ and $\mathbf{A P}(\gamma=-1)$ configuration for the Dirac electrons shown in (a) and the standard electrons shown in (b). The values of the other parameter are $\Delta=0.5, V_{2}=U_{g}=0, V_{3}=V_{g}$ and $k_{x}=k_{x_{1}}=k_{1}=2$.

by the two ferromagnetic electrodes one after another, so the phase interference will appear. As a result, this implies the existence of Fabry-Perot-like electron interference in a $\mathbf{F} / \mathbf{N} / \mathbf{F}$ tunneling based on the $\mathbf{T I}$.

\section{CONCLUSION}

In summary, we have theoretically investigated transport features of Dirac electrons on the surface of a three-dimensional TI under the modulation of a exchange field provided by an FI stripes. We find that the conductance at the $\mathbf{P}$ configuration can be much bigger than that at the AP configuration. Compared $\mathbf{P}$ with AP configuration, there exists a shift of phase which can be tuned by gate voltage. We find that the exchange field weakly affects the conductance of carriers 


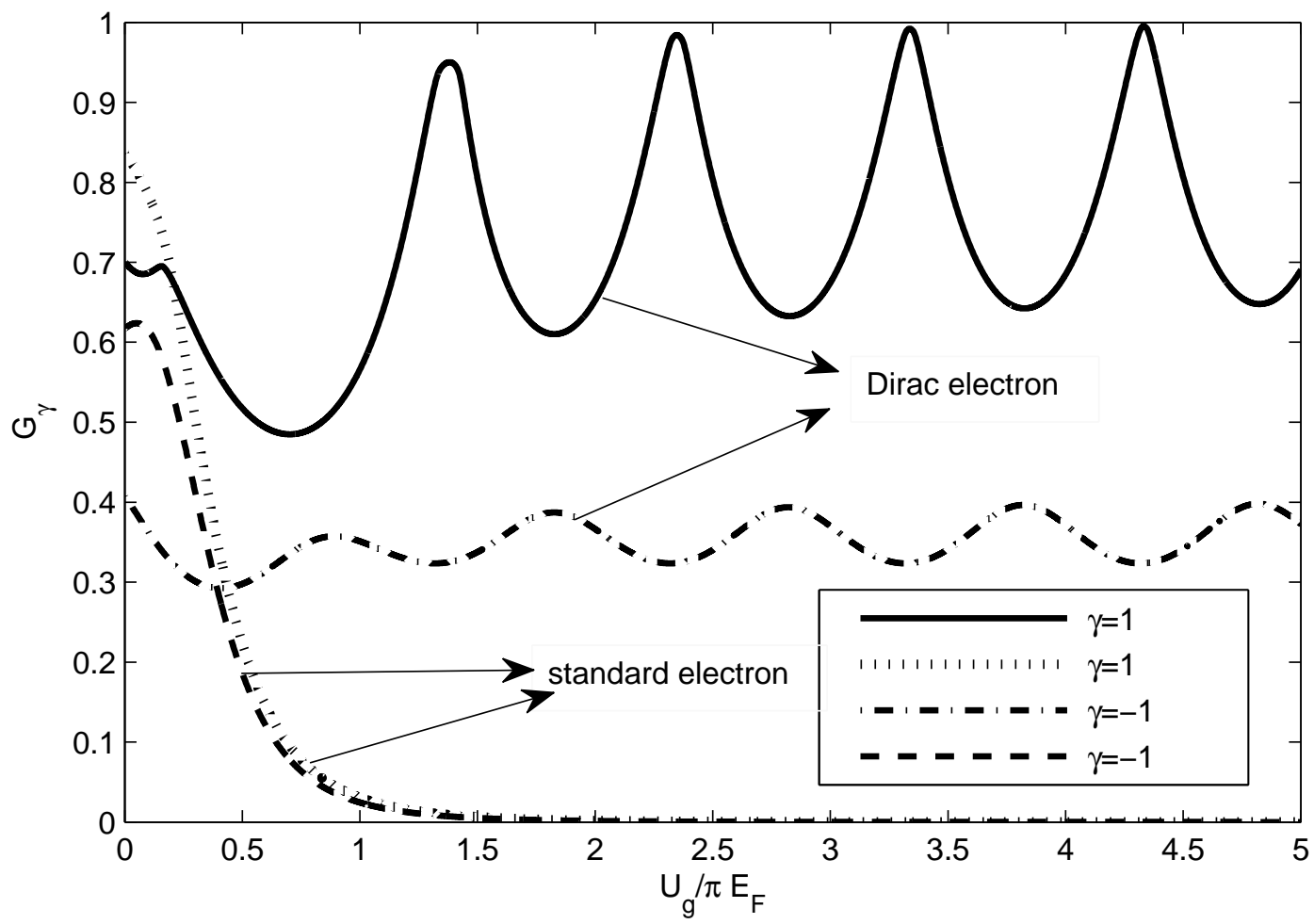

FIG. 7: Gate voltage $U_{g}$ dependence of the conductance with a $\mathbf{P}(\gamma=1)$ and $\mathbf{A P}(\gamma=-1)$ configuration for the Dirac electrons and the standard electrons. The values of the other parameter are $\eta=0.5, V_{3}=V_{g}=0$ and $E_{F}=1.0$.

for $\mathbf{P}$ configuration but can dramatically suppress the conductance of carriers for $\mathbf{A P}$ configuration. This controllable electron transport implies anomalous magnetoresistance in this topological spin valve, which may contribute to the development of spintronics .

Acknowledgments: This work was supported by the National Natural Science Foundation of China under Grants No. 10174024 and No. 10474025.

* The corresponding author, E-mail: jianhui831110@163.com

1 C. L. Kane and E. J. Mele, Phys. Rev. Lett. 95, 146802 2005; C. L. Kane and E. J. Mele, Phys. Rev. Lett. 95, 2268012005 
2 L. Fu, C. L. Kane, and E. J. Mele, Phys. Rev. Lett. 98, 106803 (2007).

3 D. Hsieh, D. Qian, L. Wray, Y. Xia, Y. Hor, R. J. Cava, and M. Z. Hasan, Nature (London) 452, 970 (2008).

4 Y. Xia, D. Qian, D. Hsieh, L. Wray, A. Pal, H. Lin, A. Bansi, D. Grauer, Y. S. Hor, R. J. Cava, M. Z. Hasan, Nature Phys. 5, 398 (2009); H. J. Zhang, C.-X. Liu, X.-L. Qi, X. Dai, Z. Fang and S.-C. Zhang, Nature Phys. 5, 438 (2009)

5 T. Eguchi, P. Gilkey, and A. Hansen, Phys. Rep. 66, 213 (1980)

6 E. I. Rashba, Sov. Phys. Solid State 2, 1109 (1960); Yu. A. Bychkov and E. I. Rashba, J. Phys. C 17, $6039(1984)$

7 D. Hsieh, Y. Xia, L. Wray, D. Qian, A. Pal, J. H. Dil, J. Osterwalder, F. Meier, G. Bihlmayer, C. L. Kane, Y. S. Hor, R. J. Cava, and M. Z. Hasan, Science 323, 919 (2009)

8 A. Nishide, A. Taskin, Y. Takeichi, T. Okuda, A. A. Kakizaki, T. Hirahara, K. Nakatsuji, F. Komori, Y. Ando, and I. Matsuda, Phys. Rev. B 81, 041309 (2010)

9 X.-L. Qi and S.-C. Zhang, Phys. Today 63, 33 (2010); J. E. Moore, Nature (London) 464, 194 (2010)

10 M. Z. Hasan and C. L. Kane, Rev. Mod. Phys. 82, 3045 ( 2010).

11 M. Z. Hasan and C. L. Kane, Rev. Mod. Phys. 82, 3045 ( 2010)

12 B. A. Bernevig and S. C. Zhang, Phys. Rev. Lett. 96, 1068022006

13 C. Wu, B. A. Bernevig, and S. C. Zhang, Phys. Rev. Lett. 96, 1064012006

14 C. Xu and J. E. Moore, Phys. Rev. B 73, 0453222006

15 L. Fu and C. L. Kane, Phys. Rev. B 74, 195312 (2006); L. Fu and C. L. Kane, Phys. Rev. B 76, 045302 (2007)

16 X.-L. Qi, T. L. Hughes, and S.-C. Zhang, Phys. Rev. B 78, 195424 (2008)

17 B. A. Bernevig, T. L. Hughes, and S. C. Zhang, Science 314, 1757 (2006)

18 M. König, S. Wiedmann, C. Brüne, A. Roth, H. Buhmann, L. Molenkamp, X.-L. Qi, and S.-C. Zhang, Science 318, 766 (2007)

19 J. E. Moore and L. Balents, Phys. Rev. B 75, 121306(R) (2007)

20 L. Fu, C. L. Kane, and E. J. Mele, Phys. Rev. Lett. 98, 106803 (2007)

21 J. C. Y. Teo, L. Fu, and C. L. Kane, Phys. Rev. B 78, 045426 (2008)

22 Chang K and Lou W K, Phys. Rev. Lett. 106206802 ( 2011) 
23 Q. Liu, C. X. Liu, C. Xu, X. L. Qi, and S. C. Zhang, Phys. Rev. Lett. 102, 156603 (2009); A. A. Burkov and D. G. Hawthorn, Phys. Rev. Lett. 105, 066802 (2010)

24 X. L. Qi, R. D. Li, J. D. Zang, and S. C. Zhang, Science 323, 1184 (2009)

25 X. L. Qi, T. L. Hughes, and S. C. Zhang, Phys. Rev. B 78, 195424 (2008)

26 T. Yokoyama, Y. Tanaka, and N. Nagaosa, Phys. Rev. B 81, 121401(R) (2010);

27 S. J. Lee, S. Souma, G. Ihm, and K. J. Chang, Phys. Rep. 394, 1 (2004)

28 M. Cerchez, S. Hugger, T. Heinzel, and N. Schulz, Phys. Rev. B 75, 035341 (2007); G. Papp and F. M. Peeters, Appl. Phys. Lett 78, 2184 (2001)

29 A. Matulis, F. M. Peeters, and P. Vasilopoulos, Phys. Rev. Lett. 72, 1518 (1994)

30 Sungjae Cho, Yung-Fu Chen and Michael S. Fuhre, Appl. Phys. Lett. 91, 123105 (2007)

31 I. Snyman and C. W. J. Beenakker, Phys. Rev. B 75, 045322 (2007)

32 Ya Zhang and Feng Zhai, Appl. Phys. Lett. 96, 172109 (2010)

33 A. R. Akhmerov, Johan Nilsson, and C.W. J. Beenakker, Phys. Rev. Lett. 102, 216404 (2009)

34 P. Adroguer, C. Grenier, D. Carpentier, J. Cayssol, P. Degiovanni, and E. Orignac, Phys. Rev. B 82, 081303(R) (2010) 